\title{
Increased Risk of Nonalcoholic Fatty Liver Disease in Individuals with High Weight Variability
}

\author{
Inha Jung ${ }^{1}$, Dae-Jeong Koo ${ }^{2}$, Mi Yeon Lee ${ }^{3}$, Sun Joon Moon ${ }^{1}$, Hyemi Kwon ${ }^{1}$, Se Eun Park ${ }^{1}$, Eun-Jung Rhee ${ }^{1}$, \\ Won-Young Lee ${ }^{1}$ \\ ${ }^{1}$ Division of Endocrinology and Metabolism, Department of Internal Medicine, Kangbuk Samsung Hospital, Sungkyunkwan \\ University School of Medicine, Seoul; ${ }^{2}$ Division of Endocrinology and Metabolism, Department of Internal Medicine, \\ Changwon Fatima Hospital, Changwon; ${ }^{3}$ Division of Biostatistics, Department of R\&D Management, Kangbuk Samsung \\ Hospital, Sungkyunkwan University School of Medicine, Seoul, Korea
}

Background: Weight loss through lifestyle modification is recommended for patients with nonalcoholic fatty liver disease (NAFLD). Recent studies have suggested that repeated loss and gain of weight is associated with worse health outcomes. This study aimed to examine the association between weight variability and the risk of NAFLD in patients without diabetes.

Methods: We examined the health-checkup data of 30,708 participants who had undergone serial examinations between 2010 and 2014. Weight variability was assessed using coefficient of variation and the average successive variability of weight (ASVW), which was defined as the sum of absolute weight changes between successive years over the 5 -year period divided by 4 . The participants were classified according to the baseline body mass index and weight difference over 4 years.

Results: On dividing the participants into four groups according to ASVW quartile groups, those in the highest quartile showed a significantly increased risk of NAFLD compared to those in the lowest quartile (odds ratio [OR], 1.89; 95\% confidence interval [CI], 1.63 to 2.19). Among participants without obesity at baseline, individuals with high ASVW showed increased risk of NAFLD (OR, 1.80; 95\% CI, 1.61 to 2.01). Participants with increased weight over 4 years and high ASVW demonstrated higher risk of NAFLD compared to those with stable weight and low ASVW (OR, 4.87; 95\% CI, 4.29 to 5.53).

Conclusion: Regardless of participant baseline obesity status, high weight variability was associated with an increased risk of developing NAFLD. Our results suggest that further effort is required to minimize weight fluctuations after achieving a desirable body weight.

Keywords: Body weight maintenance; Body weight changes; Fatty liver; Non-alcoholic fatty liver disease; Obesity; Insulin resistance

Received: 7 May 2021, Revised: 17 June 2021, Accepted: 7 July 2021

Corresponding authors: Eun-Jung Rhee

Division of Endocrinology and Metabolism, Department of Internal Medicine, Kangbuk Samsung Hospital, Sungkyunkwan University School of Medicine, 29 Saemunan-ro, Jongno-gu, Seoul 03181, Korea

Tel: +82-2-2001-2485, Fax: +82-2-2001-1588, E-mail: hongsiri@hanmail.net

Won-Young Lee

Division of Endocrinology and Metabolism, Department of Internal Medicine, Kangbuk Samsung Hospital, Sungkyunkwan University School of Medicine, 29 Saemunan-ro, Jongno-gu, Seoul 03181, Korea

Tel: +82-2-2001-2579, Fax: +82-2-2001-2049, E-mail: drlwy@hanmail.net

\section{Copyright $@ 2021$ Korean Endocrine Society}

This is an Open Access article distributed under the terms of the Creative Commons Attribution Non-Commercial License (https://creativecommons.org/ licenses/by-nc/4.0/) which permits unrestricted non-commercial use, distribution, and reproduction in any medium, provided the original work is properly cited. 


\section{INTRODUCTION}

Obesity prevalence has increased to pandemic levels over the past decades [1]. Obesity is a major public health concern because it is a known risk factor for various diseases, such as hypertension, stroke, several cancers, type 2 diabetes mellitus, and nonalcoholic fatty liver disease (NAFLD) $[2,3]$.

NAFLD is another major health challenge that has reached epidemic levels globally, ranging from simple hepatic steatosis to steatohepatitis [4]. As a consequence of the worldwide spread of obesity, the global prevalence of NAFLD is estimated at $24 \%$ [5]. The epidemiology of NAFLD varies worldwide, and it is usually parallel to the prevalence of obesity [6]. In most patients, NAFLD is closely associated with metabolic comorbidities, such as obesity and diabetes; therefore, NAFLD is considered a hepatic manifestation of metabolic syndrome [7]. The pathophysiology of NAFLD has led to several pharmacologic trials; however, none of them has yet been approved. Numerous studies have reported that weight reduction may improve hepatic steatosis and associated metabolic parameters [8]. Thus, weight loss is the primary goal of therapy and is recommended in numerous clinical guidelines for the management of NAFLD [9]. However, only a few patients achieved target weight loss and frequently failed to maintain adequate body weight in the long term [10]. Repeated patterns of weight loss and gain are called weight cycling. Recently, researchers have reported on weight cycling and its negative health consequences [11,12], and it is unclear whether weight fluctuation induces unfavorable changes in the liver. To our knowledge, few studies have been conducted to evaluate the association between weight fluctuation and the risk of NAFLD. Therefore, the aim of this study was to assess the association between weight fluctuation and NAFLD in a population without diabetes.

\section{METHODS}

\section{Source of data}

This study is part of the Kangbuk Samsung Health Study, which includes participants from a medical health screening program at the Health Promotion Center of Kangbuk Samsung Hospital, Seoul and Suwon, Korea. In Korea, it is mandatory for every employee to undergo regular health-checkup programs to enhance the early detection of disease. Most of the participants are employees and their family members, belonging to various industrial companies countrywide. The medical examinations are largely paid for by employers, and a considerable proportion of the examinees undergo examinations annually or biannually.

This study was approved by the Institutional Review Board of Kangbuk Samsung Hospital of Korea (KBSMC 2020-11019). Anonymous, unidentifiable information was used for the analysis. Therefore, the requirement for informed consent was waived.

\section{Study population and design}

Among the participants of the general health-checkup program, we selected 30,708 aged $\geq 18$ years who had undergone annual health examinations for 5 consecutive years, from January 2010 to December 2014. To examine the relationships between weight fluctuation and incident NAFLD, we excluded participants with NAFLD at baseline $(n=9,017)$. Since insulin resistance is associated with the pathophysiology of NAFLD, 1,110 participants with diabetes (defined by fasting blood glucose [FBG] level $\geq 126 \mathrm{mg} / \mathrm{dL}$, glycated hemoglobin [HbAlc] $\geq 6.5 \%$, or self-reported history of diabetes) were excluded at baseline. Individuals with positive hepatitis viral serology results, those who self-reported having a chronic liver disease, such as hepatitis or hepatocellular carcinoma, and heavy alcohol drinkers were excluded. We also excluded participants with a history of any kind of malignancy $(n=721)$ or history of medication $(n=246)$. Further, subjects with missing data were excluded $(n=2,323)$. A total of 15,340 participants were included in the final analyses. Supplemental Fig. S1 shows the process used to select study participants for this study.

\section{Anthropometric and laboratory measurement}

Data on medical history, medication use, and health-related behaviors were collected through a self-administered questionnaire. Physical measurements and serum biochemical parameters were obtained by trained staff after 12 hours of fasting. The questionnaire was based on the fourth Korea National Health and Nutritional Examination Survey [13] and the Korean version of the International Physical Activity Questionnaire short form [14]. Information on smoking and alcohol consumption was obtained using the aforementioned questionnaires. In this study, heavy alcohol consumption was defined as a daily alcohol consumption $\geq 30 \mathrm{~g}$ for men and $\geq 20 \mathrm{~g}$ for women. Regular exercise was defined as performing $>30$ minutes of moderate physical activity at least five times per week or $>20$ minutes of strenuous physical activity at least three times per week.

Body mass index (BMI) was defined as body weight in kilograms divided by the square of the height in meters squared. A BMI cut off of $25 \mathrm{~kg} / \mathrm{m}^{2}$ was used to define obesity in this Ko- 
rean study population. Systolic and diastolic blood pressures (BPs) were measured using a standardized sphygmomanometer.

$\mathrm{HbA1c}, \mathrm{FBG}$, aspartate transaminase (AST), alanine aminotransferase (ALT), total cholesterol, triglyceride, high-density lipoprotein cholesterol, and low-density lipoprotein cholesterol (LDL-C) levels were measured after 12 hours of fasting. The presence of diabetes was defined according to the following criteria: (1) having a self-reported history of diabetes, current use of an oral antidiabetic agent, or insulin use at baseline or (2) FBG level $\geq 126 \mathrm{mg} / \mathrm{dL}$ or HbA1c level $\geq 6.5 \%$. Estimated glomerular filtration rate was calculated using the Chronic Kidney Disease Epidemiology Collaboration equation [15]. Insulin resistance was assessed using the homeostatic model assessment of insulin resistance (HOMA-IR) equation [16].

\section{Measurement of weight variability}

The differences $\left(D_{1}\right)$ in body weight between 2 consecutive years were calculated by subtracting the body weight in the first year $\left(\mathrm{W}_{1}\right)$ from that in the second year $\left(\mathrm{W}_{2}\right)(\mathrm{D} 1=\mathrm{W} 2-\mathrm{W} 1)$. Subsequently, the difference was transformed into an absolute value to obtain the absolute change in body weight between the 2 years $\left(\left|\mathrm{D}_{1}\right|\right)$. We calculated the average successive variability of weight (ASVW) by adding the four $\left|\mathrm{D}_{\mathrm{y}}\right| \mathrm{s}$ and dividing the result by 4 [12]. The formula used to calculate an individual's ASVW is as follows: ASVW $=\left(\left|\mathrm{D}_{1}\right|+\left|\mathrm{D}_{2}\right|+\left|\mathrm{D}_{3}\right|+\left|\mathrm{D}_{4}\right|\right) / 4$. Body weight variability was also assessed by coefficient of variation (CV) [17].

\section{Definition of weight stability}

The stability of the body weight was defined according to the difference in body weight between the final examination $\left(\mathrm{W}_{2014}\right)$ and baseline examination $\left(\mathrm{W}_{2010}\right)$. We defined "stable weight" as $<5 \%$ weight change, "increased weight" as $\geq 5 \%$ weight gain, and "decreased weight" as $\geq 5 \%$ weight loss [18]. Thereafter, participants were divided into the following six groups according to the stability of body weight over 4 years and the ASVW: stable weight and low ASVW, stable weight and high ASVW, increased weight and low ASVW, increased weight and high ASVW, decreased weight and low ASVW, and decreased weight and high ASVW. We conducted same analysis using CV instead of ASVW.

\section{Diagnosis of nonalcoholic fatty liver disease}

The endpoint of this study was the detection of newly diagnosed NAFLD on abdominal ultrasonography (Logic Q700 MR, GE, Milwaukee, WI, USA). NAFLD was identified based on known standard criteria, including hepatorenal echo contrast, liver brightness, and vascular blurring using a $3.5-\mathrm{MHz}$ probe. Eleven experienced radiologists who were unaware of this study and were blinded to the clinical status of the subjects performed the ultrasound examinations. The inter- and intra-observer reliabilities of fatty liver diagnosis were high, with kappa statistics of 0.74 and 0.94 , respectively [19].

\section{Statistical analysis}

Continuous variables are presented as mean \pm standard deviation. Categorical variables are expressed as numbers (percentages). Right-skewed variables (i.e., HOMA-IR, AST, ALT, and alcohol intake) underwent logarithmic transformation for analysis. The Student's $t$ test for continuous variables and chi-square test for categorical variables were used to compare baseline characteristics between subjects who developed NAFLD and those who did not. The analyses were performed in the following ASVW quartile groups: $<1.13,1.13-1.625,1.625-2.33$, and $\geq 2.33 \mathrm{~kg}$. In addition, the participants were divided into the following two groups according to the median ASVW: high and low. The median ASVW was $1.625 \mathrm{~kg}$ and the median CV was $2.71 \mathrm{~kg}$. Logistic regression analysis was performed to evaluate the odds ratio (OR) of NAFLD development, according to the quartiles of weight variability after adjusting for confounding variables. The following variables were entered into logistic regression models: age, sex, FBG, systolic BP, triglyceride, LDL$\mathrm{C}$, smoking amount, alcohol intake, and frequency of vigorous exercise. Statistical significance was defined as a two-sided $P$ value $<0.05$. All statistical analyses were performed using STATA version 16.1 (StataCorp, College Station, TX, USA).

\section{RESULTS}

\section{Baseline characteristics of study participants according to the development of NAFLD after 4 years}

The characteristics of the participants at baseline are presented in Table 1. The participants' mean age was 38.1 years, and $42.5 \%$ of them were men. Among all 15,340 participants, 2,406 (15.7\%) developed NAFLD after 4 years. Those who developed NAFLD were more likely to have obesity, have higher total cholesterol and LDL-C levels, and have higher FBG levels compared to participants who did not develop NAFLD. Participants who developed NAFLD after 4 years also had higher mean HOMA-IR values than those who did not develop NAFLD $(1.32 \pm 0.72$ vs. $1.05 \pm 0.59)$. When the baseline metabolic parameters were compared according to ASVW quartiles (Supple- 
Table 1. Characteristics of Participants According to the Presence of Fatty Liver Disease

\begin{tabular}{|c|c|c|c|c|}
\hline \multirow{2}{*}{ Characteristic } & \multirow{2}{*}{$\operatorname{Total}(n=15,340)$} & \multicolumn{2}{|c|}{ Presence of fatty liver disease after 4 years } & \multirow{2}{*}{$P$ value } \\
\hline & & No $(n=12,934)$ & Yes $(n=2,406)$ & \\
\hline Age, yr & $38.1 \pm 5.8$ & $38.1 \pm 5.7$ & $38.4 \pm 6.0$ & 0.003 \\
\hline Male sex & $6,514(42.5)$ & $4,833(37.4)$ & $1,681(69.9)$ & $<0.001$ \\
\hline BMI, $\mathrm{kg} / \mathrm{m}^{2}$ & $21.9 \pm 2.6$ & $21.5 \pm 2.5$ & $23.8 \pm 2.5$ & $<0.001$ \\
\hline Waist circumference, $\mathrm{cm}$ & $77.7 \pm 7.8$ & $76.6 \pm 7.4$ & $83.5 \pm 7.0$ & $<0.001$ \\
\hline HOMA-IR & $1.1 \pm 0.6$ & $1.1 \pm 0.6$ & $1.3 \pm 0.7$ & $<0.001$ \\
\hline HbAlc, \% & $5.6 \pm 0.2$ & $5.5 \pm 0.2$ & $5.6 \pm 0.2$ & $<0.001$ \\
\hline Fasting blood glucose, $\mathrm{mg} / \mathrm{dL}$ & $92.1 \pm 7.8$ & $91.7 \pm 7.8$ & $94.1 \pm 7.7$ & $<0.001$ \\
\hline Total cholesterol, mg/dL & $188.0 \pm 31.4$ & $186.5 \pm 31.1$ & $196.3 \pm 31.9$ & $<0.001$ \\
\hline Triglyceride, mg/dL & $89.1 \pm 50.0$ & $83.8 \pm 43.2$ & $117.4 \pm 70.2$ & $<0.001$ \\
\hline HDL-C, mg/dL & $61.7 \pm 14.3$ & $63.1 \pm 14.2$ & $54.3 \pm 12.6$ & $<0.001$ \\
\hline LDL-C, mg/dL & $113.1 \pm 28.9$ & $110.9 \pm 28.4$ & $124.5 \pm 28.5$ & $<0.001$ \\
\hline $\mathrm{AST}, \mathrm{IU} / \mathrm{L}^{\mathrm{a}}$ & $19.4 \pm 9.9$ & $19.2 \pm 10.2$ & $20.5 \pm 7.4$ & $<0.001$ \\
\hline $\mathrm{ALT}, \mathrm{IU} / \mathrm{L}^{\mathrm{a}}$ & $17.4 \pm 10.7$ & $16.6 \pm 10.2$ & $21.6 \pm 12.2$ & $<0.001$ \\
\hline SBP, $\mathrm{mm} \mathrm{Hg}$ & $105.6 \pm 12.1$ & $104.7 \pm 11.9$ & $110.3 \pm 11.8$ & $<0.001$ \\
\hline Alcohol intake, $g /$ day $^{a}$ & $6.3 \pm 6.6$ & $5.9 \pm 6.4$ & $8.1 \pm 7.5$ & $<0.001$ \\
\hline Current smoker & 2,234 (14.6) & $1,569(12.1)$ & $665(27.6)$ & $<0.001$ \\
\hline Regular exercise $^{b}$ & $1,824(11.9)$ & $1,548(12.0)$ & $276(11.5)$ & 0.912 \\
\hline Obesity $^{c}$ & $1,784(11.6)$ & $1,111(8.6)$ & $673(28.0)$ & 0.177 \\
\hline
\end{tabular}

Values are expressed as mean \pm standard deviation or number $(\%)$.

BMI, body mass index; HOMA-IR, homeostasis model assessment of insulin resistance; HbA1c, glycated hemoglobin; HDL-C, high-density lipoprotein cholesterol; LDL-C, low-density lipoprotein cholesterol; AST, aspartate transaminase; ALT, alanine aminotransferase; SBP, systolic blood pressure.

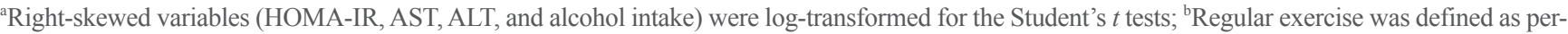

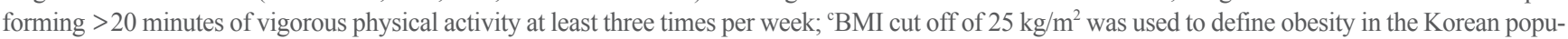
lation in this study.

mental Table S1), those in the highest ASVW quartile were younger and more obese than those in other groups. Participants in the highest ASVW quartile had worse metabolic parameters than those of participants in other groups, including FBG, HOMA-IR, total cholesterol, and LDL-C.

\section{Risk of NAFLD according to the quartiles of variability of body weight}

The proportion of participants with NAFLD increased linearly from the first to the fourth ASVW quartile $(9.3 \%, 13.1 \%$, $16.8 \%$, and $23.4 \%$; $P$ for trend $<0.001$ ) (Fig. 1). When the OR for incident NAFLD was analyzed according to ASVW and CV quartiles (Table 2), the risk of NAFLD increased linearly as ASVW and CV quartiles increased from the first to the fourth. After adjustment for various confounding variables, the ORs attenuated; however, a higher risk of NAFLD in participants with high ASVW and high CV was still observed (adjusted OR, 1.89; $95 \%$ confidence interval [CI], 1.63 to 2.19 ; and adjusted OR,

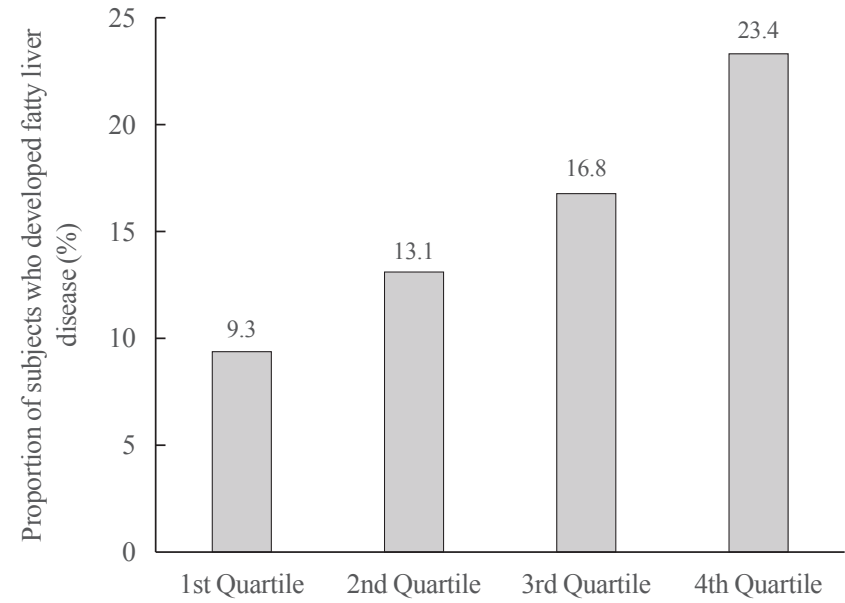

Fig. 1. The proportion of participants with nonalcoholic fatty liver disease according to quartiles of the average successive variability of body weight. Cut off value for average successive variability of weight: 1st quartile $(<1.13 \mathrm{~kg})$, 2nd quartile $(1.13-1.625 \mathrm{~kg})$, 3rd quartile $(1.625-2.33 \mathrm{~kg})$, 4th quartile $(\geq 2.33 \mathrm{~kg})$. 
Table 2. Risk of Nonalcoholic Fatty Liver Disease According to the Quartiles of Body Weight Variability

\begin{tabular}{lccc}
\hline & Model 1 & Model 2 & Model 3 \\
\hline $\begin{array}{l}\text { Quartiles of ASVW } \\
\text { 1st quartile }\end{array}$ & 1.00 (reference) & 1.00 (reference) & 1.00 (reference) \\
2nd quartile & $1.39(1.20-1.61)$ & $1.36(1.17-1.59)$ & $1.29(1.11-1.51)$ \\
3rd quartile & $1.75(1.52-2.02)$ & $1.70(1.47-1.97)$ & $1.52(1.31-1.76)$ \\
4th quartile & $2.77(2.41-3.18)$ & $2.59(2.25-2.99)$ & $1.89(1.63-2.19)$ \\
Quartiles of CV & & & $1.00($ reference) \\
1st quartile & 1.00 (reference) & 1.00 (reference) & $1.33(1.15-1.54)$ \\
2nd quartile & $1.33(1.16-1.53)$ & $1.36(1.18-1.57)$ & $1.73(1.50-2.00)$ \\
3rd quartile & $1.70(1.49-1.96)$ & $1.74(1.51-2.01)$ & $2.83(2.45-3.26)$ \\
4th quartile & $2.93(2.56-3.35)$ & $3.05(2.66-3.51)$ & \\
\hline
\end{tabular}

Values are expressed as odds ratio (95\% confidence interval). Model 1: Adjusted for age and sex; Model 2: Adjusted for Model 1+fasting blood glucose, systolic blood pressure, triglyceride, low-density lipoprotein cholesterol, smoking status, exercise, and alcohol intake; Model 3: Adjusted for Model $2+$ homeostatic model assessment of insulin resistance, glycated hemoglobin, baseline body mass index, aspartate transaminase. Cut off value for ASVW: 1st quartile $(<1.13 \mathrm{~kg})$, 2nd quartile $(1.13-1.625 \mathrm{~kg})$, 3rd quartile $(1.625-2.33 \mathrm{~kg})$, 4th quartile $(\geq 2.33 \mathrm{~kg})$; Cut off value for CV: $1 \mathrm{st}$ quartile $(<1.9 \mathrm{~kg})$, 2nd quartile (1.9-2.71 kg), 3rd quartile (2.71-3.84 kg), 4th quartile ( $\geq 3.84 \mathrm{~kg})$.

ASVW, average successive variability of weight; CV, coefficient of variation.

Table 3. Risk of NAFLD Development According to Obesity Status at Baseline and Body Weight Variability

\begin{tabular}{lcccc}
\hline & Presence of NAFLD & Model 1 & Model 2 & Model 3 \\
\hline Normal and low ASVW & $655(9.2)$ & 1.00 (reference) & 1.00 (reference) & 1.00 (reference) \\
Normal and high ASVW & $1,078(16.7)$ & $1.84(1.65-2.04)$ & $1.81(1.63-2.02)$ & $1.80(1.61-2.01)$ \\
Obese and low ASVW & $203(38.3)$ & $3.94(3.23-4.80)$ & $3.12(2.55-3.83)$ & $2.77(2.26-3.41)$ \\
Obese and high ASVW & $470(37.5)$ & $4.44(3.84-5.13)$ & $3.52(3.03-4.09)$ & $3.11(2.66-3.62)$ \\
Normal and low CV & $673(9.8)$ & $1.00($ reference) & 1.00 (reference) & $1.00($ reference) \\
Normal and high CV & $1,060(15.8)$ & $1.99(1.79-2.22)$ & $2.05(1.84-2.29)$ & $2.05(1.84-2.29)$ \\
Obese and low CV & $301(36.2)$ & $3.45(2.92-4.07)$ & $2.80(2.36-3.33)$ & $2.50(2.10-2.97)$ \\
Obese and high CV & $372(39.1)$ & $5.36(4.57-6.28)$ & $4.27(3.62-5.03)$ & $3.76(3.18-4.44)$ \\
\hline
\end{tabular}

Values are expressed as number (\%) or odds ratio (95\% confidence interval). Model 1: Adjusted for age and sex; Model 2: Adjusted for Model 1+fasting blood glucose, systolic blood pressure, triglyceride, low-density lipoprotein cholesterol, smoking status, exercise, and alcohol intake; Model 3: Adjusted for Model 2+homeostatic model assessment of insulin resistance, glycated hemoglobin, aspartate transaminase.

NAFLD, nonalcoholic fatty liver disease; ASVW, average successive variability of weight; CV, coefficient of variation.

${ }^{a}$ Body mass index cut off of $25 \mathrm{~kg} / \mathrm{m}^{2}$ was used to define obesity in the Korean population in this study.

$2.83 ; 95 \%$ CI, 2.45 to 3.26 , respectively).

\section{Risk of NAFLD development according to baseline obesity status and variability of body weight}

Table 3 presents the risk of NAFLD according to baseline obesity status and weight variability. When the participants were divided into four groups according to their obesity status and weight variability, the lowest incidence of NAFLD was observed in the non-obese and low ASVW group. When the OR for NAFLD development was analyzed in these groups, those in the non-obese and high ASVW group had higher ORs than those in the non-obese and low ASVW group (OR, 1.80; 95\% CI, 1.61 to 2.01). Similar results were observed in the analysis using CV instead of ASVW. Participants in the non-obese and high CV group showed higher risk for NAFLD than those in the non-obese and low CV group (OR, 2.05; 95\% CI, 1.84 to 2.29). Participants with obesity at baseline showed a significantly increased risk of incident NAFLD regardless of their weight variability after adjusting for confounding variables including HOMA-IR and HbAlc. 
Odds ratio $(95 \% \mathrm{Cl})$

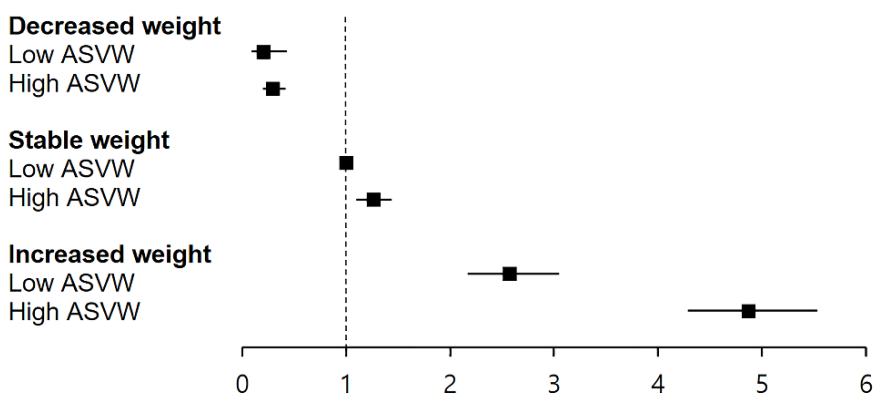

$0.20(0.09-0.43)$

$0.29(0.20-0.42)$

1 (reference)

$1.26(1.10-1.44)$

$2.57(2.17-3.05)$

4.87 (4.29-5.53)

A

Odds ratio $(95 \% \mathrm{Cl})$

Decreased weight

Low CV

High CV

Stable weight

Low CV

High CV

Increased weight

Low CV

High CV

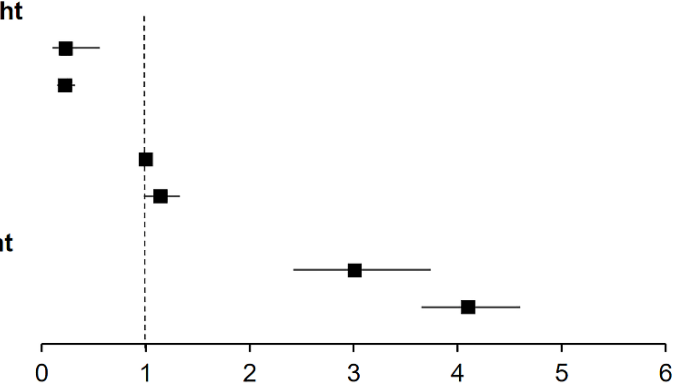

$0.23(0.10-0.56)$

$0.22(0.15-0.32)$

1 (reference)

$1.14(0.98-1.33)$

3.01 (2.43-3.74)

$4.10(3.65-4.60)$

Fig. 2. Risk of nonalcoholic fatty liver disease (NAFLD) according to the stability of body weight over 4 years and body weight variability. (A) Body weight variability was assessed by average successive variability of weight (ASVW). (B) Body weight variability was assessed by coefficient of variation (CV). CI, confidence interval.

\section{Risk of NAFLD according to the stability of body weight over 4 years and average successive variability of body weight}

When we divided participants into six groups according to the stability of body weight over 4 years and the weight variability (Fig. 2), those with a high ASVW or high CV showed higher ORs for NAFLD than those with a low ASVW or low CV. Participants with increased weight over 4 years and high ASVW showed the highest risk of NAFLD compared to those with stable weight and low ASVW (OR, 4.87; 95\% CI, 4.29 to 5.53). Participants with increased weight over 4 years and high $\mathrm{CV}$ showed higher risk for NAFLD than those with stable weight and low CV (OR, 4.10; 95\% CI, 3.65 to 4.60). Even in participants with stable weight, those with high weight variability were at risk of NAFLD compared to those with low weight variability. Among participants who lost weight over 4 years, those with a high variability of body weight showed a higher incidence of NAFLD (Supplemental Fig. S2).

\section{Risk of NAFLD in participants without transitions in obesity status from baseline to follow-up according to the variability of body weight}

We divided participants into four groups according to transitions in obesity status from baseline to follow-up as follows: nonobese to non-obese with low ASVW, non-obese to non-obese with high ASVW, obese to obese with low ASVW, and obese to obese with high ASVW. When the risk of NAFLD development was analyzed in these four groups (Table 4), participants who had obesity in succession with high ASVW had the highest risk of NAFLD, using the continuously non-obese, low ASVW group as the reference (OR, 4.85; 95\% CI, 4.11 to 5.72). Those who were continuously without obesity with high ASVW showed an increased risk of NAFLD compared to the reference group (OR, 1.39; 95\% CI, 1.22 to 1.57). Similar results were observed in the analysis using CV instead of ASVW.

\section{DISCUSSION}

Obesity with subsequent insulin resistance plays a key role in the pathogenesis of NAFLD. Therefore, previous studies have suggested that weight reductions may induce resolution of steatohepatitis or improve hepatic fibrosis [20]. However, the majority of individuals who lose weight have difficulty maintaining the reduced weight for an extended period of time [21]. To the best of our knowledge, no study has identified the relationship between weight variability in healthy people and the risk of 
Table 4. Risk of NAFLD in Participants without Transitions between Obesity Status from Baseline to Follow-up According to the Variability of Body Weight

\begin{tabular}{lcccc}
\hline & Presence of NAFLD & Model 1 & Model 2 & Model 3 \\
\hline Non-obese to non-obese, low ASVW & $547(8.1)$ & 1.00 (reference) & 1.00 (reference) & 1.00 (reference) \\
Non-obese to non-obese, high ASVW & $637(11.7)$ & $1.42(1.25-1.60)$ & $1.39(1.23-1.58)$ & $1.39(1.22-1.57)$ \\
Obese to obese, low ASVW & $200(41.8)$ & $5.21(4.23-6.42)$ & $4.20(3.39-5.21)$ & $3.76(3.02-4.67)$ \\
Obese to obese, high ASVW & $457(43.7)$ & $6.66(5.70-7.78)$ & $5.43(4.62-6.39)$ & $4.85(4.11-5.72)$ \\
Non-obese to non-obese, low CV & $559(8.6)$ & 1.00 (reference) & 1.00 (reference) & $1.00($ reference) \\
Non-obese to non-obese, high CV & $625(10.9)$ & $1.54(1.36-1.75)$ & $1.58(1.39-1.79)$ & $1.58(1.39-1.80)$ \\
Obese to obese, low CV & $296(39.1)$ & $4.50(3.78-5.36)$ & $3.68(3.07-4.41)$ & $3.30(2.74-3.96)$ \\
Obese to obese, high CV & $361(47.1)$ & $8.79(7.4-10.46)$ & $7.32(6.13-8.76)$ & $6.52(5.44-7.82)$ \\
\hline
\end{tabular}

Values are expressed as number (\%) or odds ratio (95\% confidence interval). Model 1: Adjusted for age and sex; Model 2: Adjusted for Model 1+fasting blood glucose, systolic blood pressure, triglyceride, low-density lipoprotein cholesterol, smoking status, exercise, and alcohol intake; Model 3: Adjusted for Model 2+homeostatic model assessment of insulin resistance, glycated hemoglobin, aspartate transaminase.

NAFLD, nonalcoholic fatty liver disease; ASVW, average successive variability of weight; CV, coefficient of variation.

${ }^{a}$ Body mass index cut off of $25 \mathrm{~kg} / \mathrm{m}^{2}$ was used to define obesity in the Korean population in this study.

NAFLD, especially in a large cohort.

In the present study, we found that fluctuations in body weight over 4 years were significantly associated with increased NAFLD risk. Individuals with greater ASVW and greater CV were associated with a higher incidence and higher risk of NAFLD compared to those with reference-weight variability. Among participants without obesity and even among those who constantly had no obesity, those who had large fluctuations in body weight had a higher risk of NAFLD after adjusting for confounding variables. As expected, the increased risk of NAFLD became greater among individuals who gained weight over 4 years with high ASVW or high CV.

Many studies have suggested that fluctuations in body weight may have negative health outcomes. The Framingham Heart Study reported increased mortality and morbidity due to coronary heart disease in subjects with highly variable body weights [12]. In a Korean nationwide cohort study, high variabilities in body weight were associated with higher risks of cardiovascular disease and all-cause mortality [22]. We provided evidence that weight cycling was associated with the development of diabetes [23], and the findings of the present study are in line with the results of previous studies.

The mechanisms behind the relationship between weight fluctuation and NAFLD are unclear; nevertheless, several hypotheses have been proposed to explain this phenomenon. Insulin resistance and hyperinsulinemia resulting from weight fluctuation are considered key components in the pathogenesis of NAFLD [24]. A plausible mechanism responsible for insulin re- sistance is that of changes in fat distribution and increased percentage of body fat followed by weight cycling. Previous studies reported that weight cycling may cause a redistribution of body fat to the upper body compartment, strengthening the risk of metabolic diseases, including fatty liver disease [25-27]. This alteration in body composition is associated with insulin resistance and induces alterations in fatty-acid metabolism [28,29]. In our cohort of young and middle-aged participants without diabetes, participants in the highest ASVW quartile had higher HOMA-IR values than in those from other quartiles (Supplemental Table S1).

Our findings support the hypothesis that weight fluctuation has unfavorable effects on various health outcomes. However, our study had several limitations. First, NAFLD was diagnosed using abdominal ultrasonography and not liver biopsy. Although liver biopsy is the gold standard method for the diagnosis of fatty liver, it is invasive and unsuitable as a screening tool, especially in a large-scale cohort study. Recent studies have demonstrated that validated NAFLD predictive models overcame the limitation of ultrasonography-based diagnoses [30]. Second, previous researches on weight variability as a predictor of NAFLD are limited. Therefore, the appropriate interval for measuring weight variability has not been determined. Since we used data from annual health check-up program, we could not calculate short-term weight variability, which might be related with the pathogenesis of NAFLD. The lack of consensus about the formula of weight variability is another limitation of this study. Obese individuals are more likely to experience severe 
weight cycling [31]. We calculated weight variability using ASVW and CV with raw weight based on previous studies. However, raw weight and BMI doesn't reflect the actual body fat composition [32]. Additional studies using variability of percentage body fat ( $\%$ Fat) should be performed in the future.

Also, our study was unable to distinguish intentional weight change from unintentional weight change. We excluded participants with malignancy and those with a history of certain medication to minimize the possible effects of unintentional weight change. However, we did not consider participants' emotional distress, their psychological status or presence of eating disorders. We could not completely rule out the possibility of selection bias and unknown behavioral factors affecting body weight.

Moreover, our results were derived from a database of relatively healthy young and middle-aged Koreans who regularly participated in a health-checkup program. Those who regularly receive a health-checkup are likely to have healthier lifestyles involving good diets. Both diet and daily calorie intake are important factors that result in weight change and subsequent NAFLD; therefore, the lack of these data is another limitation of our study. However, we observed associations between weight fluctuations and increased risk of NAFLD consistently after adjustment for health-related habits, including smoking status, alcohol consumption, and frequency of regular exercise. Additional studies using data with diet information should be performed in multiple institutions and in different ethnicities in the future. Finally, the cross-sectional design of our study is limited in its ability to determine causality. Longitudinal prospective studies should be conducted in the future.

Despite these limitations, our study has several strengths. First, this study has a large sample size of relatively young and healthy participants without diabetes, providing the independent contribution of weight fluctuation on the development of NAFLD. Second, we classified study subjects in various ways to evaluate the effects of weight variability and weight stability. Third, we performed sensitivity analyses to prove the robustness of our results. A sensitivity analysis in which we evaluated NAFLD outcomes using the fatty liver index yielded similar results (Supplemental Table S2) [33].

In this large cohort study of young and middle-aged adults without diabetes, weight fluctuation was significantly associated with an increased risk of developing NAFLD. Weight reduction through diet and lifestyle changes is recommended for the prevention and management of NAFLD. Once a desirable body weight is achieved, further effort is required to minimize weight fluctuations and maintain a healthy weight.

\section{CONFLICTS OF INTEREST}

No potential conflict of interest relevant to this article was reported.

\section{ACKNOWLEDGMENTS}

The authors acknowledge the efforts of the Department of R\&D Management at Kangbuk Samsung Hospital, Korea, in editing figures and tables.

\section{AUTHOR CONTRIBUTIONS}

Conception or design: I.J., E.J.R., W.Y.L. Acquisition, analysis, or interpretation of data: D.J.K., M.Y.L., S.J.M. Drafting the work or revising: I.J., H.K., S.E.P., E.J.R., W.Y.L. Final approval of the manuscript: I.J., D.J.K., M.Y.L., S.J.M., H.K., S.E.P., E.J.R., W.Y.L.

\section{ORCID}

Inha Jung https://orcid.org/0000-0001-8561-8544

Eun-Jung Rhee https://orcid.org/0000-0002-6108-7758

Won-Young Lee https://orcid.org/0000-0002-1082-7592

\section{REFERENCES}

1. World Health Organization. Obesity and overweight [Internet]. Geneva: WHO; 2020 [cited 2021 Jul 23]. Available from: https://www.who.int/news-room/fact-sheets/detail/ obesity-and-overweight.

2. Lavie CJ, Milani RV, Ventura HO. Obesity and cardiovascular disease: risk factor, paradox, and impact of weight loss. J Am Coll Cardiol 2009;53:1925-32.

3. Li L, Liu DW, Yan HY, Wang ZY, Zhao SH, Wang B. Obesity is an independent risk factor for non-alcoholic fatty liver disease: evidence from a meta-analysis of 21 cohort studies. Obes Rev 2016;17:510-9.

4. Angulo P. Nonalcoholic fatty liver disease. N Engl J Med 2002;346:1221-31.

5. Younossi ZM, Koenig AB, Abdelatif D, Fazel Y, Henry L, Wymer M. Global epidemiology of nonalcoholic fatty liver disease-Meta-analytic assessment of prevalence, incidence, and outcomes. Hepatology 2016;64:73-84.

6. Younossi Z, Anstee QM, Marietti M, Hardy T, Henry L, Eslam M, et al. Global burden of NAFLD and NASH: 
trends, predictions, risk factors and prevention. Nat Rev Gastroenterol Hepatol 2018;15:11-20.

7. Nemes K, Aberg F. Interpreting lipoproteins in nonalcoholic fatty liver disease. Curr Opin Lipidol 2017;28:355-60.

8. Musso G, Gambino R, Cassader M, Pagano G. A meta-analysis of randomized trials for the treatment of nonalcoholic fatty liver disease. Hepatology 2010;52:79-104.

9. European Association for the Study of the Liver (EASL); European Association for the Study of Diabetes (EASD); European Association for the Study of Obesity (EASO). EASLEASD-EASO clinical practice guidelines for the management of non-alcoholic fatty liver disease. Obes Facts 2016;9:65-90.

10. Promrat K, Kleiner DE, Niemeier HM, Jackvony E, Kearns $\mathrm{M}$, Wands JR, et al. Randomized controlled trial testing the effects of weight loss on nonalcoholic steatohepatitis. Hepatology 2010;51:121-9.

11. Lissner L, Odell PM, D'Agostino RB, Stokes J 3rd, Kreger BE, Belanger AJ, et al. Variability of body weight and health outcomes in the Framingham population. N Engl J Med 1991; 324:1839-44.

12. Bangalore S, Fayyad R, Laskey R, DeMicco DA, Messerli FH, Waters DD. Body-weight fluctuations and outcomes in coronary disease. N Engl J Med 2017;376:1332-40.

13. Yoon YS, Oh SW, Baik HW, Park HS, Kim WY. Alcohol consumption and the metabolic syndrome in Korean adults: the 1998 Korean National Health and Nutrition Examination Survey. Am J Clin Nutr 2004;80:217-24.

14. Oh JY, Yang YJ, Kim BS, Kang JH. Validity and reliability of Korean version of International Physical Activity Questionnaire (IPAQ) short form. J Korean Acad Fam Med 2007; 28:532-41.

15. Levey AS, Stevens LA, Schmid CH, Zhang YL, Castro AF 3rd, Feldman HI, et al. A new equation to estimate glomerular filtration rate. Ann Intern Med 2009;150:604-12.

16. Matthews DR, Hosker JP, Rudenski AS, Naylor BA, Treacher DF, Turner RC. Homeostasis model assessment: insulin resistance and beta-cell function from fasting plasma glucose and insulin concentrations in man. Diabetologia 1985;28:412-9.

17. Kim JA, Lee JS, Chung HS, Roh E, Lee YB, Hong SH, et al. Impact of visit-to-visit fasting plasma glucose variability on the development of type 2 diabetes: a nationwide population-based cohort study. Diabetes Care 2018;41:2610-6.

18. Kim YH, Kim SM, Han KD, Son JW, Lee SS, Oh SW, et al. Change in weight and body mass index associated with allcause mortality in Korea: a nationwide longitudinal study. J Clin Endocrinol Metab 2017;102:4041-50.
19. Kim CW, Yun KE, Jung HS, Chang Y, Choi ES, Kwon MJ, et al. Sleep duration and quality in relation to non-alcoholic fatty liver disease in middle-aged workers and their spouses. J Hepatol 2013;59:351-7.

20. Vilar-Gomez E, Martinez-Perez Y, Calzadilla-Bertot L, Torres-Gonzalez A, Gra-Oramas B, Gonzalez-Fabian L, et al. Weight loss through lifestyle modification significantly reduces features of nonalcoholic steatohepatitis. Gastroenterology 2015;149:367-78.

21. Sarlio-Lahteenkorva S, Rissanen A, Kaprio J. A descriptive study of weight loss maintenance: 6 and 15 year follow-up of initially overweight adults. Int J Obes Relat Metab Disord 2000;24:116-25.

22. Kim DH, Nam GE, Han K, Kim YH, Park KY, Hwang HS, et al. Variabilities in weight and waist circumference and risk of myocardial infarction, stroke, and mortality: a nationwide cohort study. Endocrinol Metab (Seoul) 2020;35:933-42.

23. Rhee EJ, Cho JH, Kwon H, Park SE, Park CY, Oh KW, et al. Increased risk of diabetes development in individuals with weight cycling over 4 years: the Kangbuk Samsung Health Study. Diabetes Res Clin Pract 2018;139:230-8.

24. Hill AJ. Does dieting make you fat? Br J Nutr 2004;92 Suppl 1:S15-8.

25. Wallner SJ, Luschnigg N, Schnedl WJ, Lahousen T, Sudi K, Crailsheim K, et al. Body fat distribution of overweight females with a history of weight cycling. Int J Obes Relat Metab Disord 2004;28:1143-8.

26. Prentice AM, Jebb SA, Goldberg GR, Coward WA, Murgatroyd PR, Poppitt SD, et al. Effects of weight cycling on body composition. Am J Clin Nutr 1992;56(1 Suppl):209S$16 \mathrm{~S}$.

27. Cereda E, Malavazos AE, Caccialanza R, Rondanelli M, Fatati G, Barichella M. Weight cycling is associated with body weight excess and abdominal fat accumulation: a cross-sectional study. Clin Nutr 2011;30:718-23.

28. Sea MM, Fong WP, Huang Y, Chen ZY. Weight cycling-induced alteration in fatty acid metabolism. Am J Physiol Regul Integr Comp Physiol 2000;279:R1145-55.

29. Rhee EJ, Choi JH, Yoo SH, Bae JC, Kim WJ, Choi ES, et al. The association of unintentional changes in weight, body composition, and homeostasis model assessment index with glycemic progression in non-diabetic healthy subjects. Diabetes Metab J 2011;35:138-48.

30. Kozakova M, Palombo C, Eng MP, Dekker J, Flyvbjerg A, Mitrakou A, et al. Fatty liver index, gamma-glutamyltransferase, and early carotid plaques. Hepatology 2012;55:1406- 
15.

31. Field AE, Manson JE, Laird N, Williamson DF, Willett WC, Colditz GA. Weight cycling and the risk of developing type 2 diabetes among adult women in the United States. Obes Res 2004;12:267-74.

32. Prentice AM, Jebb SA. Beyond body mass index. Obes Rev
2001;2:141-7.

33. Bedogni G, Bellentani S, Miglioli L, Masutti F, Passalacqua M, Castiglione A, et al. The fatty liver index: a simple and accurate predictor of hepatic steatosis in the general population. BMC Gastroenterol 2006;6:33. 